\title{
DOA Estimation with Non-Circular Reduced Dimension PM Algorithm for linear array Zheng Wang $^{1}$, Lv Weihua ${ }^{1,2}$, Zhang Xiaofei ${ }^{1,3}$
}

1. College of Electronic and Information Engineering, Nanjing University of Aeronautics and Astronautics, Nanjing, China, 210016

2. Luoyang Optoelectro technology development center, Luoyang, China, 471000

3. National Mobile Communications Research Laboratory, Southeast University, China, 210096

Email: fei_zxf@163.com

Keywords: Array signal processing, DOA, Non-Circular signal, Reduced Dimension PM

\begin{abstract}
Propagator Method (PM) algorithm is a common method for the estimation of direction of arrival (DOA). In this paper, we propose a reduced dimension PM (RD-PM) algorithm for non-circular signal with linear array. We reduce the dimension of the pseudo spectrum function so that we need only one dimensional searching, which decreases the computational complexity. And the exploiting of the noncircular property improves the accuracy of estimation. Extensive simulations illustrate the effectiveness and improvement of our algorithm.
\end{abstract}

\section{Introduction}

The estimation of direction of arrival (DOA) is one of the fundamental problem in array signal processing and is very important for various engineering applications such as radar, sonar, acoustics, wireless communication. After the notion of non-circular signal was proposed, exploiting the non-circular property to improve the spatial spectrum estimation has become the research hotspot in signal processing, which shows some excellent advantages, such as more detectable signals and higher accuracy. Many signals in communication system are non-circular signals, such as the Amplitude Modulation (AM), the Binary Phase Shift Keying (BPSK) signals [1-2].

In [3], Haardt proposed a unitary-ESPRIT (Estimating of Signal Parameters via Rotational Invariance Techniques) algorithm for the non-circular signals which obtained the real valued matrix to estimate the DOA by unitary transformation. This algorithm improved the degree of freedom (DOF) and the resolution. In [4], Tayem and Kwon proposed a conjugate ESPRIT algorithm which improved the DOF and the resolution by constructing a new covariance matrix.

We proposed a RD-PM algorithm for non-circular signals which only needs one dimensional searching and no eigenvalue decomposition of the covariance matrix. Our algorithm has a low computational complexity and a similar performance with the standard PM algorithm.

The reminder of this paper is organized as follows. In Section 2, we present the signal model of non-circular signals. In Section 3, we describe the detailed derivation of RD-PM algorithm for non-circular signals. Simulation results are presented in Section 4 and we conclude this paper in Section5.

Notations: Lower-case (upper-case) boldface symbols denote vectors (matrices). $(\bullet)^{T},(\bullet)^{H},(\bullet)^{-1}$, $(\bullet)^{+}$denote the transpose, the conjugate transpose, the inverse and the pseudo inverse, respectively. $E(\bullet)$ denotes the expectation operator.

\section{Non-circular signals}

Signals can be distinguished by non-circular rate of the signal $E\left[s^{2}(t)\right]=\rho e^{-j \phi} E\left[s(t) s^{*}\right]=\rho e^{-j \phi} \sigma_{s}^{2}$, where $\phi$ is the non-circular phase of the signal and $\rho$ is the non-circular rate of the signal. The non-circular signals satisfy $0<\rho \leq 1$ and it is called maximum 
non-circular rate signal when $\rho=1$ specifically. For the maximum non-circular rate signal $s(t)=s_{R}(t) e^{-j \phi}$ where $s_{R}(t)$ is the real valued signal with zero initial phase and $\phi$ is the non-circular phase.

The signal vector can be represented as

$$
\begin{aligned}
& \mathbf{S}(t)=\boldsymbol{\Phi S}_{R}(t), \\
& {\left[s_{1}(t), s_{2}(t), \cdots, s_{K}(t)\right]^{T}=\boldsymbol{\Phi}\left[s_{R 1}(t), s_{R 2}(t), \cdots, s_{R K}(t)\right]^{T},}
\end{aligned}
$$

where $\boldsymbol{\Phi}=\operatorname{diag}\left\{e^{-j \phi_{1}}, e^{-j \phi_{2}}, \cdots, e^{-j \phi_{K}}\right\}$ and $\phi_{1}, \phi_{2}, \cdots, \phi_{K}$ are the non-circular phases of $s_{1}(t), s_{2}(t), \cdots, s_{K}(t)$ respectively.

\section{Data model}

We assume that there are $K$ uncorrelated narrowband signals impinging on the uniform linear array equipped with $M$ sensors. The inter-element spacing is half of the wavelength. We also assume that sources are far away from the array. The noise is additive independent identically distributed Gaussian with zero mean and variance $\sigma_{n}^{2}$, which is independent of signals. We denote the elevation angles of sources as $\boldsymbol{\theta}=\left[\theta_{1}, \theta_{2}, \cdots, \theta_{K}\right]^{T}$, where $\theta_{k}$ is the elevation angle of the $k$ th source.

The array output can be modeled as

$$
\mathbf{X}(t)=\mathbf{A S}(t)+\mathbf{N}(t), t=1,2, \cdots, L,
$$

where $\mathbf{X}(t)=\left[\mathbf{x}_{1}(t), \mathbf{x}_{2}(t), \cdots, \mathbf{x}_{M}(t)\right], \mathbf{A}=\left[\mathbf{a}\left(\theta_{1}\right), \mathbf{a}\left(\theta_{2}\right), \cdots, \mathbf{a}\left(\theta_{K}\right)\right] \in C^{M \times K}$ is the steering matrix, $\mathbf{a}\left(\theta_{k}\right)=\left[1, e^{-j \mu_{k}}, \cdots, e^{-j(M-1) \mu_{k}}\right]^{T}, \mu_{k}=2 \pi d \sin \theta_{k} / \lambda, \mathbf{S}(t)=\left[s_{1}(t), s_{2}(t), \cdots, s_{K}(t)\right]^{T}$ is the signal vector and $L$ is the number of snapshots. $\mathbf{N}(t)=\left[n_{1}(t), n_{2}(t), \cdots, n_{K}(t)\right]^{T}$ is the noise vector.

According to the non-circular property of the signals, we construct the extended array output as

$$
\mathbf{Y}(t)=\left[\begin{array}{c}
\mathbf{X}(t) \\
\mathbf{X}^{*}(t)
\end{array}\right]=\left[\begin{array}{c}
\mathbf{A}(\theta) \mathbf{\Phi} \\
\mathbf{A}^{*}(\theta) \mathbf{\Phi}^{*}
\end{array}\right] \mathbf{S}_{R}(t)+\left[\begin{array}{c}
\mathbf{N}(t) \\
\mathbf{N}^{*}(t)
\end{array}\right]=\mathbf{B S}_{R}(t)+\mathbf{N}^{\prime}
$$

where the extended steering matrix is

$$
\mathbf{B}=\left[\begin{array}{c}
\mathbf{A}(\theta) \mathbf{\Phi} \\
\mathbf{A}^{*}(\theta) \mathbf{\Phi}^{*}
\end{array}\right]=\left[\mathbf{b}\left(\theta_{1}, \phi_{1}\right), \mathbf{b}\left(\theta_{2}, \phi_{2}\right), \cdots, \mathbf{b}\left(\theta_{K}, \phi_{K}\right)\right] \text { and } \mathbf{b}(\theta, \phi)=\left[\begin{array}{c}
\mathbf{a}(\theta) e^{-j \phi} \\
\mathbf{a}^{*}(\theta) e^{j \phi}
\end{array}\right]
$$

And the covariance matrix of the extended array output is

$$
\mathbf{R}_{Y}=E\left[\mathbf{Y}(t) \mathbf{Y}^{H}(t)\right]=\left[\begin{array}{c}
\mathbf{A}(\theta) \mathbf{\Phi} \\
\mathbf{A}^{*}(\theta) \mathbf{\Phi}^{*}
\end{array}\right] \mathbf{R}_{S}\left[\begin{array}{c}
\mathbf{A}(\theta) \mathbf{\Phi} \\
\mathbf{A}^{*}(\theta) \boldsymbol{\Phi}^{*}
\end{array}\right]^{H}+\sigma_{n}^{2} \mathbf{I}_{2 M},
$$

where $\mathbf{R}_{S}=E\left[\mathbf{S}_{R}(t) \mathbf{S}_{R}^{H}(t)\right]$ is the covariance matrix of signal $\mathbf{S}_{R}(t)$.

In practice, the covariance matrix is replaced by the following sample average

$$
\hat{\mathbf{R}}_{Y}=\frac{1}{L} \mathbf{Y}(t) \mathbf{Y}^{H}(t)
$$

Partition the extended steering matrix as

$$
\mathbf{B}=\left[\begin{array}{l}
\mathbf{B}_{1} \\
\mathbf{B}_{2}
\end{array}\right]
$$

where $\mathbf{B}_{1} \in C^{K \times K}$ and $\mathbf{B}_{2} \in C^{(2 M-K) \times K}$.

The propagation operator $\mathbf{P} \in C^{K \times(2 M-K)}$ is defined as

$$
\mathbf{P}^{H} \mathbf{B}_{1}=\mathbf{B}_{2},
$$




$$
\left[\mathbf{P}^{H}, \quad-\mathbf{I}_{2 M-K}\right] \mathbf{B}=\mathbf{Q}^{H} \mathbf{B}=\mathbf{0},
$$

\section{PM algorithm}

Partition the extended covariance matrix $\mathbf{R}_{Y}$ as

$$
\mathbf{R}_{Y}=[\mathbf{G}, \mathbf{H}],
$$

where $\mathbf{G} \in C^{2 M \times K}$ and $\mathbf{H} \in C^{2 M \times(2 M-K)}$.

Propagation operator can be estimated by

$$
\hat{\mathbf{P}}=\left(\mathbf{G}^{H} \mathbf{G}\right)^{-1} \mathbf{G}^{H} \mathbf{H},
$$

Then we can get

$$
\hat{\mathbf{Q}}^{H}=\left[\hat{\mathbf{P}}^{H},-\mathbf{I}_{2 M-K}\right],
$$

In order to introduce $\hat{\mathbf{Q}}$ into the projection operator of the noise subspace, we replace it with its orthogonalized matrix $\hat{\mathbf{Q}}_{0}=\hat{\mathbf{Q}}\left(\hat{\mathbf{Q}}^{H} \hat{\mathbf{Q}}\right)^{-1 / 2}$.

According to (10), we can also obtain $\hat{\mathbf{Q}}_{0}^{H} \mathbf{b}(\theta, \phi)=\mathbf{0}$.

Then we can construct the pseudo spectrum function

$$
f_{P M}(\theta, \phi)=\mathbf{b}^{H}(\theta, \phi) \mathbf{Q}_{0} \mathbf{Q}_{0}^{H} \mathbf{b}(\theta, \phi),
$$

By two dimensional searching, we can obtain the minimal value of (14) and get the estimation of DOA and non-circular phase.

\section{Reduced dimension transformation}

Partition $\hat{\mathbf{Q}}_{0}$ as

$$
\hat{\mathbf{Q}}_{0}=\left[\begin{array}{c}
\hat{\mathbf{Q}}_{1} \\
\hat{\mathbf{Q}}_{2}
\end{array}\right]
$$

where $\hat{\mathbf{Q}}_{1} \in C^{M \times(2 M-K)}$ and $\hat{\mathbf{Q}}_{2} \in C^{M \times(2 M-K)}$.

Reconstruct (5),

$$
\mathbf{b}(\theta, \phi)=\left[\begin{array}{l}
\mathbf{a}(\theta) e^{-j \phi} \\
\mathbf{a}^{*}(\theta) e^{j \phi}
\end{array}\right]=\left[\begin{array}{cc}
\mathbf{a}(\theta) & \mathbf{O} \\
\mathbf{O} & \mathbf{a}^{*}(\theta)
\end{array}\right]\left[\begin{array}{l}
e^{-j \phi} \\
e^{j \phi}
\end{array}\right],
$$

Then, (14) can be represented as

$$
f_{P M}(\theta, \phi)=\left[\begin{array}{c}
e^{-j \phi} \\
e^{j \phi}
\end{array}\right]^{H}\left[\begin{array}{ll}
\mathbf{a}^{H}(\theta) \hat{\mathbf{Q}}_{1} \hat{\mathbf{Q}}_{1}^{H} \mathbf{a}(\theta) & \mathbf{a}^{H}(\theta) \hat{\mathbf{Q}}_{1} \hat{\mathbf{Q}}_{2}^{H} \mathbf{a}^{*}(\theta) \\
\mathbf{a}^{T}(\theta) \hat{\mathbf{Q}}_{2} \hat{\mathbf{Q}}_{1}^{H} \mathbf{a}(\theta) & \mathbf{a}^{T}(\theta) \hat{\mathbf{Q}}_{2} \hat{\mathbf{Q}}_{2}^{H} \mathbf{a}^{*}(\theta)
\end{array}\right]\left[\begin{array}{c}
e^{-j \phi} \\
e^{j \phi}
\end{array}\right]=\mathbf{q}^{H} \mathbf{M} \mathbf{q},
$$

Take partial $\phi$ derivative of (17) and let $\frac{\partial f_{P M}(\theta, \phi)}{\partial \phi}=0$, we can obtain

$$
e^{-j 2 \phi}= \pm \frac{M_{12}}{\left\|M_{12}\right\|}
$$

Then take partial $\theta$ derivative of (17) and let $\frac{\partial f_{P M}(\theta, \phi)}{\partial \theta}=0$. We will find (17) has the minimum value when (18) takes the positive value.

Combine (17) and (18) to construct the pseudo spectrum function

$$
f_{N C-R D-P M}(\theta)=M_{11}+M_{22}-2\left\|M_{12}\right\|,
$$

By only one dimensional searching, we can obtain the minimal value of (19) and get the estimation of DOA and non-circular phase. 


\section{Advantages and complexity}

Advantages

(1) The algorithm needs no eigenvalue decomposition of the covariance matrix and needs only one dimensional searching which improves the computational efficiency.

(2) The algorithm has better performance compared with the standard PM algorithm.

(3) The algorithm can achieve the joint estimation of DOA and the non-circular phase and can be paired automatically.

Complexity

The complexity of our algorithm is $O\left(4 M^{2} L+4 M^{2} K+2 M K^{2}+K^{3}+4 M^{2} K P+8 M^{2} P\right)$.

\section{Simulation results}

Suppose $K=3$ sources impinging on the array located at $\left(\theta_{1}, \phi_{1}\right)=\left(10^{\circ}, 15^{\circ}\right),\left(\theta_{2}, \phi_{2}\right)=\left(20^{\circ}, 25^{\circ}\right)$ and $\left(\theta_{3}, \phi_{3}\right)=\left(30^{\circ}, 35^{\circ}\right)$. The root mean square error (RMSE) is defined as the performance metric

$$
R M S E=\sqrt{\frac{1}{S K} \sum_{s=1}^{S} \sum_{k=1}^{K}\left(\alpha_{k}-\hat{\alpha}_{k, s}\right)^{2}},
$$

where $S$ denotes the times of Monte-Carlo simulations and $\hat{\alpha}_{k, s}$ is the estimation of the $k$ th angle $\alpha_{k}$ for the sth trial $(S=100)$.

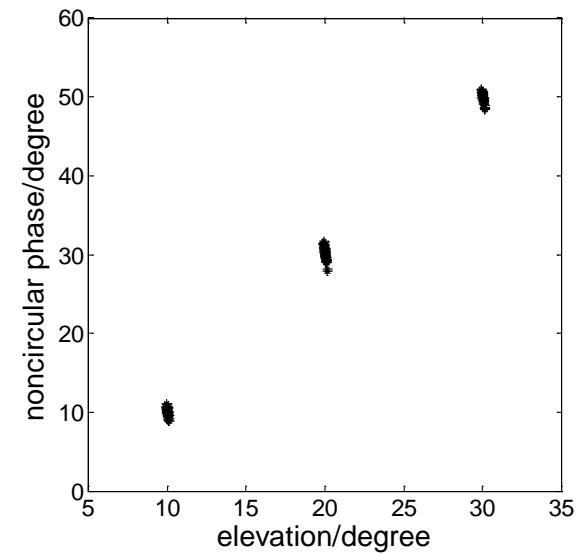

a

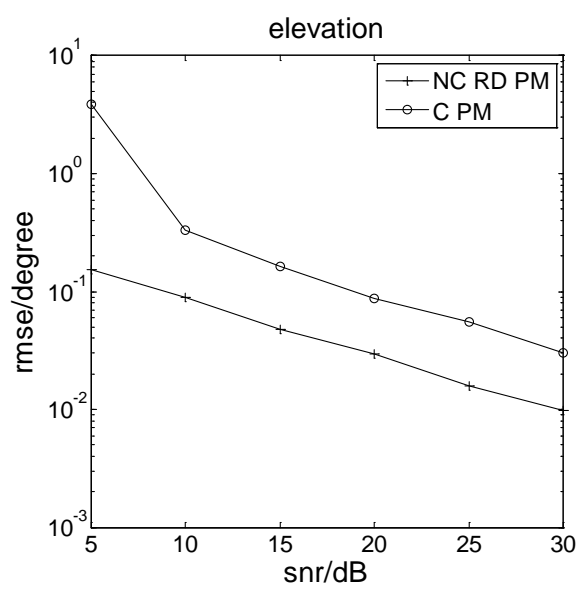

b

Fig.1 Simulation result of DOA estimation and non-circular phase.

Fig.2 Comparison of NC-RD-PM and standard C-PM.

The Fig. 1 shows the simulation results when $S N R=15 d B$ where $M=8, L=100$.

Firstly, we study the RMSE performance of the NC-RD-PM and the standard PM under different SNRs $(M=8, L=100)$ as shown in Fig.2. It is clearly indicated that it can achieve better performance than the standard PM algorithm. Secondly, we change the number of sensors $(L=100)$. It is clearly indicated that the performance of NC-RD-PM algorithm is getting better with $M$ increasing because of diversity gain as shown in Fig.3. Finally, we change the number of snapshots $L(M=8)$. It is clearly indicated as shown in Fig.4 that it is getting better with $L$ increasing. 


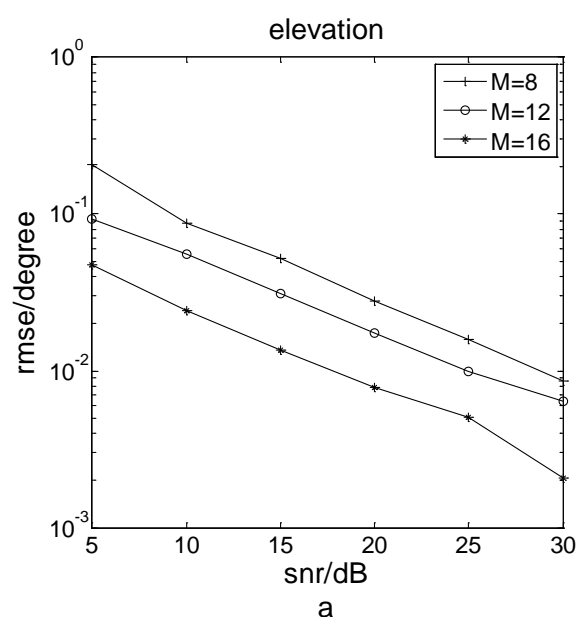

Fig.3 Performance of NC-RD-PM with different number of sensors.

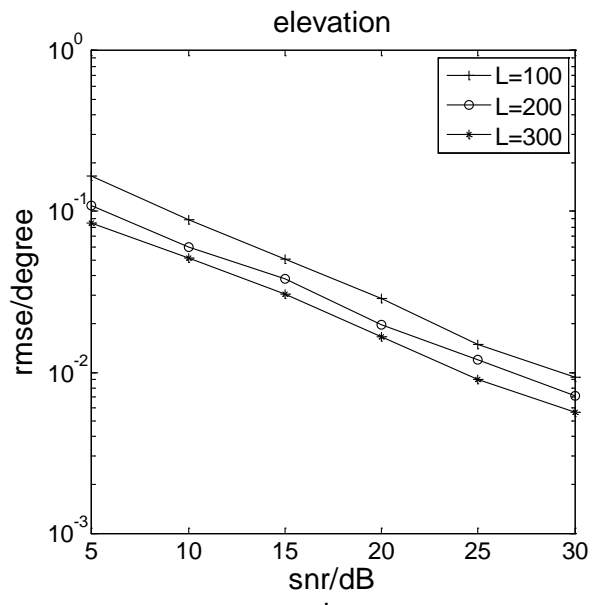

Fig.4 Performance of NC-RD-PM with different snapshots.

\section{Summary}

In this paper, we propose a RD PM algorithm for non-circular signals with linear array. The RD PM algorithm for non-circular signals needs no eigenvalue decomposition and only one dimensional searching so that it has a low computational complexity. Extensive simulations illustrate the effectiveness and improvement of our algorithm.

\section{References}

[1] Barbaresco F, Chevalier P, "Non-circularity exploitation in signal processing overview and application to radar,” IET Waveform Diversity \& Digital Radar Conference, pp:1-6, 2009.

[2] Liu Jian, Huang Zhitao, Zhou Yiyu, “A survey of direction finding for non-circular signals,” Electronic countermeasure, no.5, pp:44-49, 2007.

[3] Haardt M, Romer F, “Enhancements of unitary ESPRIT for non-circular sources,” International Conference on Acoustics, Speech, and Signal Processing, ICASSP, pp:101-104, 2004.

[4] Tayem N, Kwon H M, “Conjugate ESPRIT ( C-ESPRIT ),” IEEE Transaction on Antennas and Propagator, vol.52, no.10, pp:2618-2624, 2004.

[5] Zheng Chundi, Feng Dazheng, Zhou Yi, et al, "A real value algorithm of ESPRIT via exploitation of non-circular sources property," Journal of Electronics and Information Technology, vol.30, no.1, pp:130-133, 2008.

[6] Zheng Chundi, Feng Dazheng, Lei Ge, "DOA estimation algorithm for non-circular sources using real value algorithm," Journal of Data Acquisition and Processing, vol.24, no.2, pp:193-197, 2009.

[7] H. Abeida, J. P. Delmas, "MUSIC-Like Estimation of Direction of Arrival for Noncircular Sources,” IEEE Transaction on Signal Processing, vol.54, no.7, pp:2678-2690, 2006.

[8] Gounon P, Adnet C, Galy J, “Localization angulaire de signaux non circulaires,” Traitement du Signal, vol.15, no.1, pp:17-23, 1998.

[9] Zhang Xiaofei, Zhang Licen, Chen Weiyang, et al, “Computationally efficient DOA estimation for MIMO array using propagator method and the reduced-dimension transformation,” Journal of Data Acquisition and Processing, vol.29, no.3, pp:372-377, 2014. 